\title{
Q-compensation for High Resolution Seismic Imaging
}

Jerry M. Harris and Tieyuan Zhu

Department of Geophysics, Stanford University, USA

Copyright 2014, SBGf - Sociedade Brasileira de Geofísica

Este texto foi preparado para a apresentação no VI Simpósio Brasileiro de Geofísica, Porto Alegre, 14 a 16 de outubro de 2014. Seu conteúdo foi revisado pelo Comitê Técnico do VI SimBGf, mas não necessariamente representa a opinião da SBGf ou de seuS associados. É proibida a reprodução total ou parcial deste material para propósitos comerciais sem prévia autorização da SBGf

\begin{abstract}
Q-compensated imaging combines attenuation $\left(Q^{-1}\right)$ estimation with attenuation-compensated reverse time migration (Q-RTM). We review the approach (theory and algorithm), and present results from a field application of $Q$-compensated imaging using borehole seismic data. The goal of Q-compensated imaging is to restore pre-stack amplitude loss, improve signal bandwidth, and improve pre-stack balance of amplitude and phase in an effort to improve stack coherency and subsequently image resolution. To this end, we developed and tested a consistent approach for $Q$ estimation and forward-time and reverse-time simulation for waves in constant $Q$ media. This approach incorporates robust and practical algorithms for Q-compensated imaging. The approach is tested on a crosswell field dataset from west Texas, USA where improved spatial resolution and improved spatial continuity can be observed in the Q-compensated image.
\end{abstract}

\section{Introduction}

The goal of borehole seismic surveys is to gain a better understanding of heterogeneity near and between boreholes. Borehole approaches seek high spatial resolution for reservoir characterization and monitoring of reservoir production processes. Previous studies have shown that seismic attenuation can vary significantly in reservoir and non-reservoir formations, an observation that can result in reduced resolution when attenuation is not considered in traditional imaging approaches such as CDP and reverse time migration.

Seismic attenuation affects both amplitude and phase of seismic signals. The effects of attenuation are dispersive with frequency and can result in reduced stack coherency and subsequently reduced spatial resolution from the stacked image. Ad hoc processing methods that attempt to compensate for attenuation provide some improvement, but often lack consistency with the physics of wave propagation in attenuating media, i.e., dispersion.

\section{Methodology for Q-compensated Imaging}

The general processing workflow for Q-compensated imaging is diagrammed in Figure 1 for borehole seismic surveys. The methodology relies on the integration of three main steps that are listed here in the reverse order of their occurrence in the workflow: (a) Q-RTM: provides a robust algorithm for forward- and reverse-time simulation of wave propagation in attenuating media.

(b) Wavefield and waveform processing: a sequence of preprocessing algorithms, e.g., filters, to prepare the dataset for Q-RTM imaging.

(c) Q-Tomography: requires a robust approach for quantitatively estimating velocity and attenuation needed by $Q$-RTM.

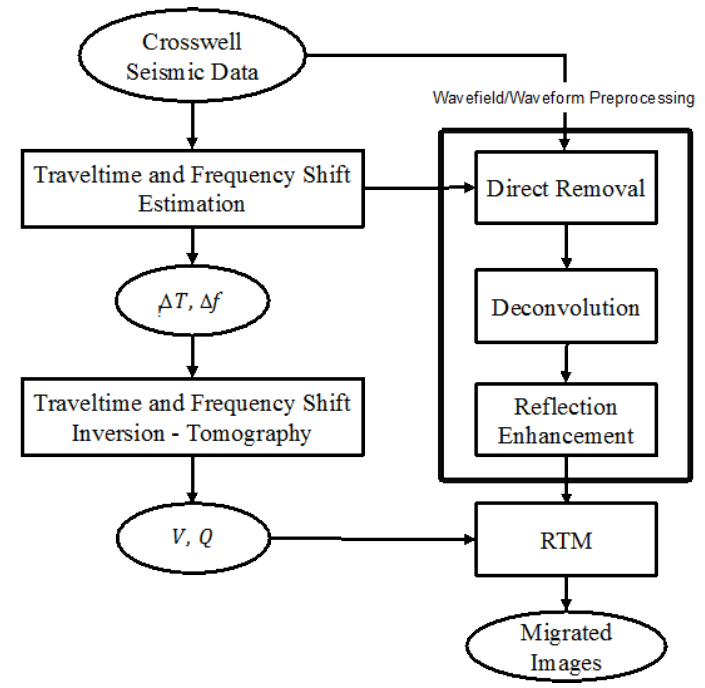

Figure 1 - General processing workflow for borehole seismic data.

Each of the three steps listed above is important to Qcompensated imaging. Nevertheless, various methods of preprocessing and $Q$-tomography exist already. The enabling technology still needed comes in Step (a), QRTM. Zhu and Harris (2014a) have formulated a wave equation simulation algorithm that partially decouples amplitude loss/gain and normal dispersion in smoothly varying media with constant $Q$ dispersion

$$
\frac{1}{c_{0}^{2}} \frac{\partial^{2} p}{\partial t^{2}}=\nabla^{2} p+\left[\eta\left(-\nabla^{2}\right)^{\gamma+1}-\nabla^{2}\right] p+\tau \frac{\partial}{\partial t}\left(-\nabla^{2}\right)^{\gamma+1 / 2} p
$$

Eqn. 1 is a formulation for viscoacoustic waves in a medium with constant $Q$. This formulation is especially useful because it partially separates dispersion and amplitude loss/gain in terms represented by the RHS terms of eqn. (1). Eqn. 1 can be used for both forward and the backward wave propagation as required by QRTM. Note too that the medium's $Q$ is in terms represented by fractional Laplacian $\gamma$ operators. For details, see Zhu and Harris (2014a).

Eqn. (1) applies to "smoothly" varying heterogenous media. Even though it is not exact, it does closely approximate dispersion in a constant $Q$ medium as 
illustrated in Figure 2 with the comparisons of the numerical simulations, theory, and laboratory data. Moreover, the quality of fit to the dispersion theory can be adjusted over a limited band (lower or higher frequencies) by selection of a reference frequency, here set at $100 \mathrm{~Hz}$ to model the higher frequencies used in borehole seismic surveys. Sample shot simulations from Eqn.1 with and without attenuation are shown in Figure 3.
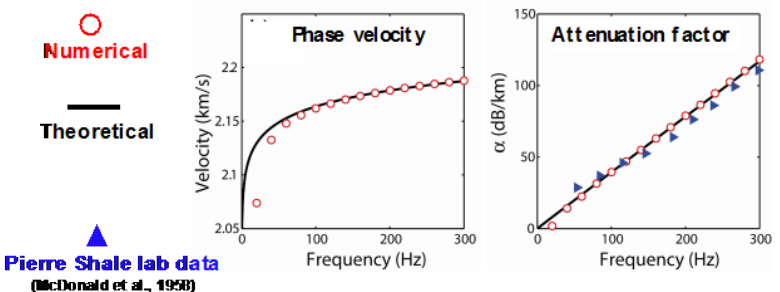

(uchonald et at, 19:3)

Figure 2 - Comparison of velocity and attenuation dispersion for a homogeneous constant $Q$ medium (Zhu, 2014c).
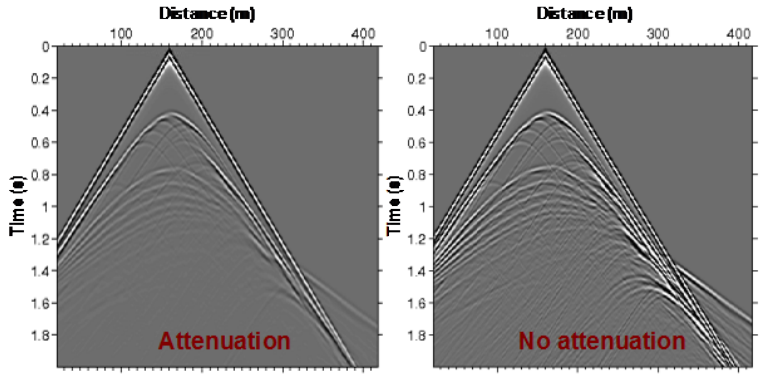

Figure 3 - Comparison of velocity and attenuation dispersion for a homogeneous constant $Q$ medium (Zhu, 2014c).

Wavefield and waveform preprocessing (step (b)) includes a sequence of spatial and temporal filters that are needed to separate the complex assemblage of wave types observed in borehole datasets, e.g. $P$ waves, $S$-waves, and $C$-waves. Together these filters seek to enhave primary reflections, remove multiples, and generate a dataset that meets the assumptions used by the $Q$-RTM algorithm.

Finally, step (c), travel-time and frequency-shift tomography is used in a joint inversion for velocity and attenuation, respectively. Previous Q-tomography studies (Quan and Harris, 1997) have shown that spatial variations in seismic attenuation can be significant. Attenuation tomography is similar to velocity tomography except the input data are frequency shifts estimated from the centroid frequency of spectrum of the direct arriving waveform. Quan and Harris (1997) introduced the frequency-shift method for the estimation of seismic attenuation. In the field data example that follows, we use a boundary-preserving joint inversion algorithm for velocity and attenuation recently developed (Zhu and Harris, 2013). In this algorithm, both travel-times and frequency-shifts are input to the joint inversion.

\section{Results with Synthetic Data}

We tested $Q$-compensated imaging with simulated data generated for a synthetic model developed by BP (Billette and Brandsberg-Dahl, 2004). The model includes a high attenuation zone (gas chimney with low $\mathrm{Q}$ value) above the imaging target at and below about $1 \mathrm{~km}$ depth. The velocity and attenuation models are shown in Figures 4a-4b. 2-D surface seismic data were simulated using a viscoacoustic algorithm (staggered grid pseudospectral method) with a standard linear solid model for the attenuation (Zhu, et al., 2013). The Q-compensated image was made using the fractional Laplacian algorithm discussed above, i.e., eqn. (1). Comparison of the Q-compensated image with the conventional RTM image (with no compensation) demonstrates the improved spatial resolution with $Q$ compensation. The improved resolution includes better location of the structure as well improved recovery of the reflectivity (Zhu, et al., 2014d).
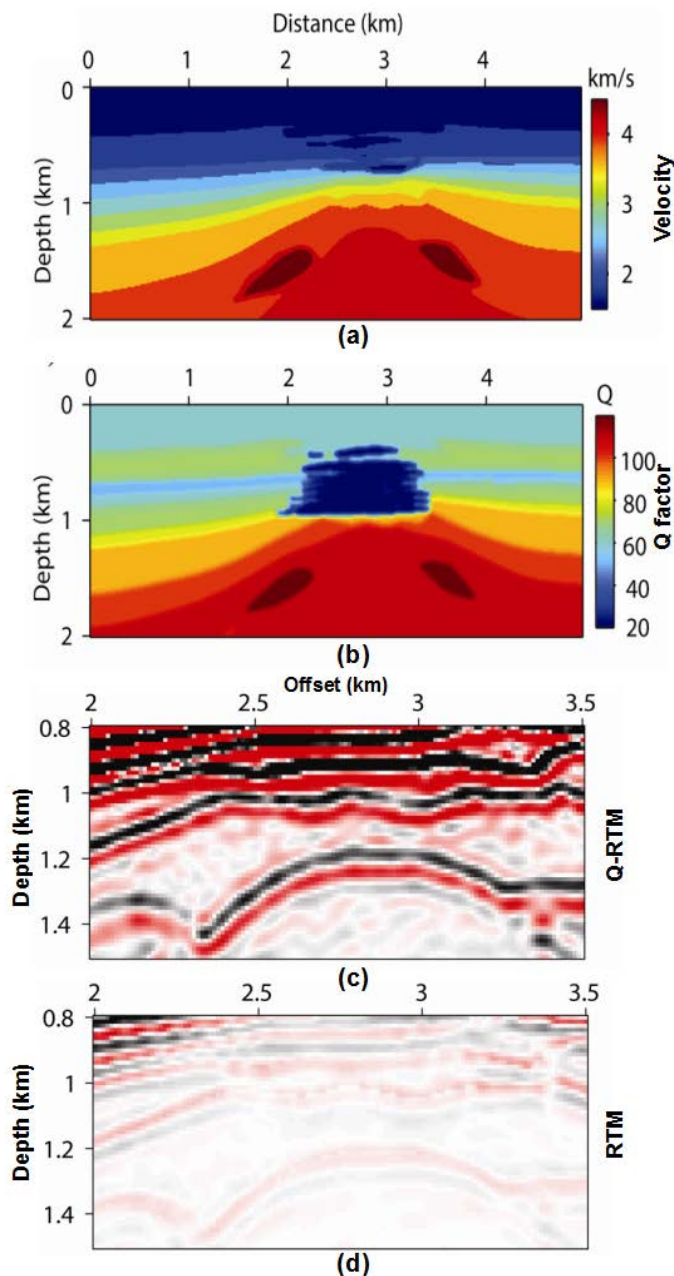

Figure 4 - Surface seismic synthetic tests: (a) P-wave velocity model; (b) P-wave $Q$ model; (c) Results of $Q$ RTM; (d) Results of RTM (Zhu, 2014e).

\section{Results with Field Data}

We tested Q-compensated imaging with data from a crosswell field survey recorded in west Texas, USA. 
The target of the survey was a carbonate mound that was identified in one well (B) but not detected in a nearby nearby well (A). All the steps outlined in the processing workflow (Figure 1) were performed for the field dataset, including the wavefield processing. The resulting images are displayed in Figure 5, one with no attenuation compensation and another with $\mathrm{Q}$ compensation. The images show a remarkable improvement of $Q$ compensation. Each RTM image is displayed with a color underlay of the velocity image, obtained from tomography, for comparison of the RTM reflectivity with velocity variations.

Figure $5 a$ shows the image obtained with traditional acoustic RTM and no attenuation compensation. This image provides reasonable stratigraphic information (for example, the reflectors between depths of 8400 feet and 8700 feet) but also displays a lack of resolution and loss of amplitude in the target area between 8700 and 8850 feet. The low Q of the reservoir zone has attenuated the reflected signals that pass through the reservoir area.

Figure $5 b$ depicts the image obtained by $Q$ compensated imaging, where attenuation (no shown) was estimated with velocity using the joint algorithm of Zhu and Harris (2013). The location of the reservoir are well inferred from the low velocity zone and the slightly dipping structures near Well B between depths of 8700 and $9000 \mathrm{ft}$.

The most striking difference between the two images is the fine scale structure that can be seen in the $Q$ compensated image but absent in the traditional image without compensation, especially inside and below the reservoir zone. The flat layers appear to truncate the dipping structure. The transition from left-dipping to flat features suggest delineation of the reservoir (Figure $5 b)$. Vertical resolution is clearly better and reflection continuity is much improved as can be seen at depths between 8500 and 8700 fee for example with the strong reflectors above the reservoir zone.

\section{Discussion and Conclusions}

The compensation of amplitude loss and velocity dispersion caused by attenuation is important for highresolution imaging. Moreover, wave equation based compensation is effective in balancing amplitudes for signals that have traversed paths of significantly different lengths as well as for paths that have experienced significantly different amount of amplitude loss due to spatial varying rates of attenuation. Amplitude restoration alone does not correct for dispersion caused by attenuation, thus the benefit achieved by the wave equation process of reverse time migration.

Tests with field data indicate that compensation for attenuation improves the image in regions where low $\mathrm{Q}$ has reduced amplitudes. Compensation also improves continuity in areas where high attenuation is less evident. Therefore, attenuation compensation not only restores amplitude loss at high frequencies, it also improves pre-stack coherence of signals presumably by making small adjustments to the velocity caused by normal dispersion in attenuating media. Improvement is evident even when the model for attenuation is not known, as we demonstrated by creating a synthetic dataset using a different model for attenuation dispersion than was used in the RTM imaging algorithm.

We used a wave equation formulation that is especially well suited for reverse time simulation. The formulation maintains the appropriate relationships of amplitude loss in reverse time propagation that is needed to compensate for attenuation experienced in forward time propagation.

In order to compensate for attenuation in RTM imaging, it is necessary to estimate the spatially varying rate of attenuation from the dataset. Q tomography based on frequency-shift data is useful in estimating consistent attenuation rates. We used a method of inversion where traveltime data and frequency shift data are jointly inverted for velocity and attenuation, respectively.

\section{Acknowledgements}

The authors thank Chevron for providing the field dataset used in this study.

\section{References}

Billette, F. J., and S. Brandsberg-Dahl, 2004, The 2004 $\mathrm{BP}$ velocity benchmark: 67th Annual International Conference and Exhibition, EAGE, Extended Abstracts, B305.

McDonald, F. J., F. A. Angona, R. L. Mills, R. L. Sengbush, R. G. Van Nostrand, and J. E. White, 1958, Attenuation of shear and compressional waves in Pierre shale: Geophysics, 23, 421-439.

Quan, Y., and Harris, J. M., 1997, Seismic attenuation tomography using the frequency shift method: Geophysics, 62, 895-905.

Zhu, T., J. M. Carcione, and J. M. Harris, 2013, Approximating constant-Q seismic propagation in the time domain: Geophysical prospecting, 61, 931-940, DOI: 10.1111/1365-2478.12044.

Zhu, T., and J. M. Harris, 2013, Application of boundary-preserving seismic inversion to delineate reservoir boundaries in Western Texas: Extended Abstract, 2013 SEG Annual Meeting.

Zhu, T. and Harris, J. M., 2014a, Modeling acoustic wave propagation in heterogeneous attenuating media using decoupled fractional Laplacians: Geophysics, 79, no.3, S165-S174.

Zhu, T. and Carcione, J. M., 2014b, Theory and modeling of constant-Q P- and S-waves using fractional spatial derivatives: Geophysical Journal International, 196, 1787-1795, doi:10.1093/gji/ggt483.

Zhu, T., 2014c, Seismic modeling, inversion, and imaging in attenuating media. Ph.D. thesis, Department of Geophysics, Stanford University

Zhu, T., J. M. Harris, and B. Biondi, 2014d, Qcompensated reverse time migration: Geophysics, 79, no.3, S77-S87. 


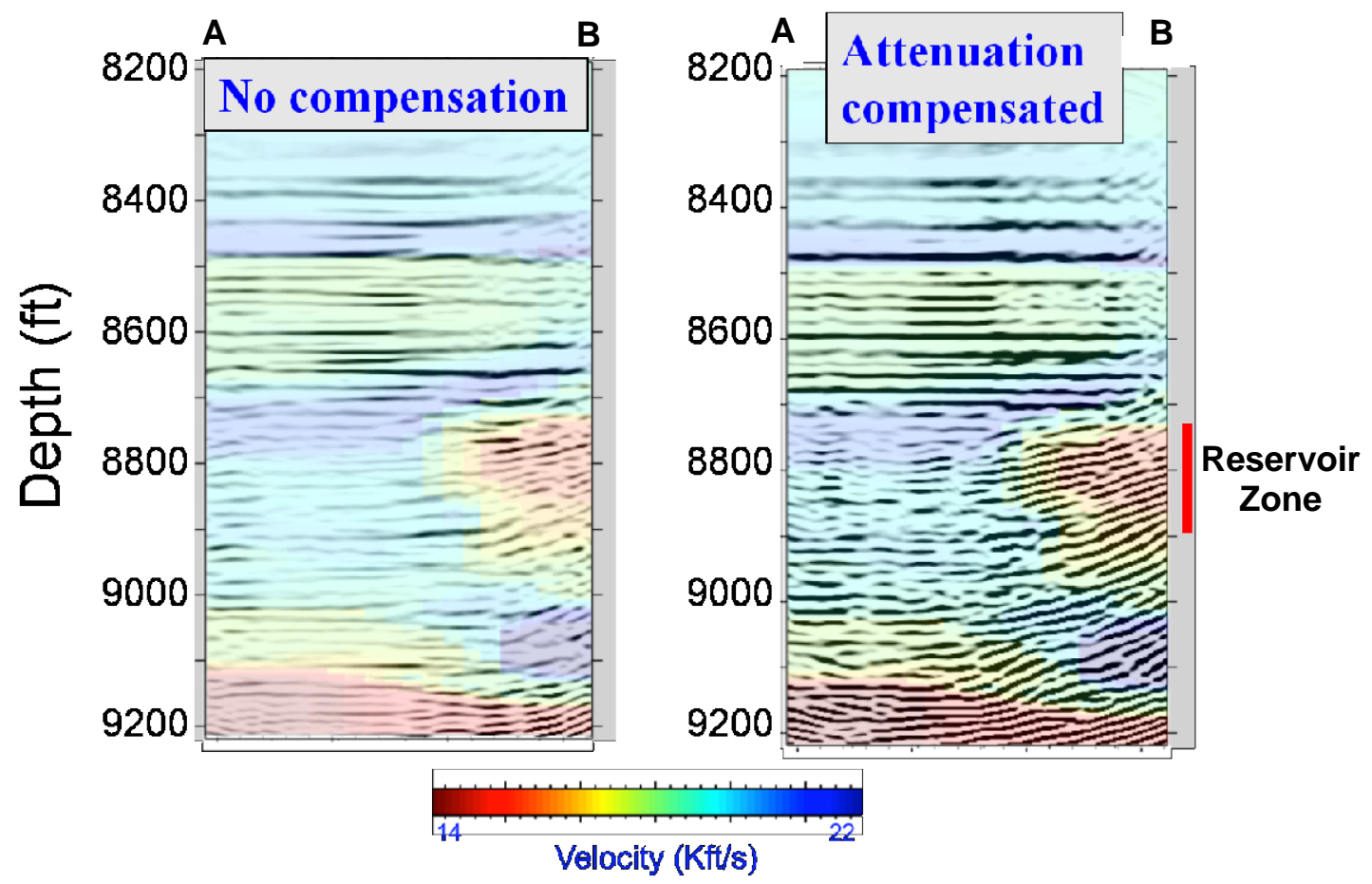

Figure 5: Seismic reflection images (a) by conventional RTM and (b) by Q-RTM overlaid on the estimated tomographic velocity. The target is the low velocity reservoir detected in Well B between 8700 feet and 8850 feet. (Zhu et al, 2014e). 